\title{
Uma Revisão Sistemática da Literatura para Investigação de Estratégias de Ensino Colaborativo
}

\author{
Simone Erbs da Costa ${ }^{1}$, Edilson Hipólito da Silva ${ }^{2}$, Leandro Otávio Córdova Vieira ${ }^{1}$, \\ Carla Diacui M. Berkenbrock ${ }^{1}$
}

\author{
${ }^{1}$ Universidade do Estado de Santa Catarina (UDESC) \\ Joinville - SC - Brasil \\ ${ }^{2}$ Instituto Federal de Santa Catarina (IFSC) \\ Jaraguá do Sul - SC - Brasil \\ si.gen@terra.com.br, edilsonhipolito@gmail.com, \\ leandro@whap.com.br, carla.berkenbrockeudesc.br
}

\begin{abstract}
This paper presents a systematic review investigating the state of the art of collaborative learning between teachers. The searches were conducted in the digital libraries of the ACM and IEEE Xplore in the selected period of 20092015 and were reviewed 591 papers. Here we highlight the results obtained and the methodologies used in the evaluations. The results demonstrate that the strategies in collaborative learning among teachers are still not considered aspects of educational cooperation, restricting the collaboration factors geared to the needs of students or the construction of teaching materials, which are managed individually and not so collaborative. We also try to check trends, strategies and limitations related to collaborative learning.
\end{abstract}

Resumo. Este artigo apresenta uma revisão sistemática que investiga o estado da arte do ensino colaborativo entre professores. As buscas foram realizadas nas bibliotecas digitais da ACM e IEEE Xplore. Foram revisados 591 artigos no período selecionado de 2009 a 2015. Neste artigo são destacados os resultados obtidos, bem como as metodologias utilizadas nas avaliações. Os dados demonstram que nas estratégias no ensino colaborativo entre professores ainda não são considerados os aspectos de colaboração de ensino, restringindo-se aos fatores de colaboração voltados às necessidades dos alunos ou na construção de material didático, os quais são geridos individualmente e não de forma colaborativa. A pesquisa também visa verificar tendências, estratégias e limitações por meio dos resultados identificados.

\section{Introdução}

O desenvolvimento das atividades relacionadas com a aprendizagem colaborativa é frequentemente defendido no meio acadêmico. Uma das razões para essa conduta é o seu potencial de promover uma aprendizagem mais ativa por meio do estímulo ao pensamento crítico [Torres 2015]. O desdobramento de capacidades de interação, negociação de informações e resolução de problemas, também são fatores relevantes.

Métodos de aprendizagem colaborativa podem tornar os alunos mais responsáveis por sua formação, conduzindo a uma aquisição do conhecimento com maior autonomia 
[Torres 2015]. Com isso, o processo de ensino-aprendizagem não está mais centrado na figura do professor e o aluno exerce um novo papel, onde o professor cria contextos e ambientes adequados para que o aluno possa desenvolver suas habilidades sociais e cognitivas de modo criativo, na interação em sala.

Neste sentido destaca-se a importância do compartilhamento de experiências entre professores. Esse compartilhamento pode favorecer o desenvolvimento da destreza na análise crítica, na resolução de problemas e na tomada de decisões [Nono and MIZUKAMI 2001].

O trabalho colaborativo multidisciplinar pode enriquecer a prática pedagógica, pois proporciona uma visão mais abrangente sobre as dificuldades enfrentadas e envolve um maior número de pessoas responsáveis pelo processo de escolarização dos alunos [Rabelo 2012]. [Pugach and Johnson 1989] salientam a importância de se realizar parcerias colaborativas na educação. Segundo [Damiani 2008], este cenário de colaboração não tem acontecido e os professores continuam trabalhando individualmente, indicando que esta tendência parece não ter mudado.

Alguns autores [Engeström 1994, Fullan et al. 2000, Thurler and Wolff 2001] descrevem a profissão docente como solitária. Já [Fullan et al. 2000] sugerem que o isolamento docente tem raízes em fatores como a arquitetura das escolas, a estrutura dos seus horários, a sobrecarga de trabalho e a própria história da profissão docente. Segundo [Pugach and Johnson 1989], o maior problema no trabalho colaborativo entre os professores são os mesmos abandonarem sua posição de perito, e compartilhar seu planejamento, medos, necessidades e potenciais com outros professores. Para [Gately and Gately 2001], o ideal seria que os professores pudessem compartilhar seu trabalho educativo, sem hierarquias, onde as responsabilidades seriam de todos os atuantes com aqueles educandos, visando o compartilhamento de planejamentos, as intervenções, monitorias, avaliações e replanejamento.

Neste trabalho foi realizado uma Revisão Sistemática da Literatura (RSL) afim de identificar o estado da arte acerca do ensino colaborativo. O estudo considerou trabalhos em inglês publicados nas bibliotecas digitais da ACM e IEEE Xplore, no período selecionado de 2009 a 2015. A revisão busca verificar iniciativas onde os professores colaboram entre si na troca ou na melhoria de experiências de ensino-aprendizagem.

O restante do trabalho está organizado da seguinte forma: A seção 2 descreve os trabalhos correlatos. A seção 3 descreve a revisão sistemática da literatura realizada. Já as seções 4 e 5 descrevem, respectivamente, a análise dos resultados obtidos por meio da revisão e as discussões sobre esses resultados. Finalmente, a seção 6 descreve as conclusões obtidas até o momento, bem como aponta os próximos passos desta pesquisa.

\section{Trabalhos correlatos}

Esta seção apresenta alguns trabalhos diretamente relacionados com a pesquisa descrita no presente artigo.

[Sa'don et al. 2014] observam a propagação de plataformas Massive Open Online Courseware (MOOC). De acordo com os autores, apesar do crescimento da literatura relacionada com MOOC, há poucos trabalhos dedicados a revisar e avaliar sistematicamente esse conteúdo produzido. Via mineração de dados, os autores encontraram mais 
de 400 artigos publicados na área. Avaliaram e sintetizaram esse material, e identificaram a possibilidade de mapeamento de tendências de pesquisa, estratégias e limitações para a utilização destas plataformas.

Segundo [Sa'don et al. 2016], as iniciativas inovadoras que incentivam a disseminação do conhecimento precisam de uma estrutura de negócio sólida para a sustentabilidade dos cursos como MOOC. A proposta impacta as partes interessadas, as instituições renomadas e os usuários finais ao acesso de educação de qualidade.

[Lin and Lin 2010], ao notarem as reformas educacionais e curriculares em Taiwan, bem como a diversidade de condições de absorção de tais reformas por parte dos docentes, experimentaram uma metodologia colaborativa (E-Teacher Community Competition - ETCC) por meio da formação de equipes entre professores. A plataforma foi desenvolvida pela Educational of Hualien County (EBHC). Os autores estimularam a produção de conteúdos colaborativos entre os membros. Ao longo de 13 semanas, os autores perceberam que o ETCC se tornou uma ferramenta de incentivo e também um novo modelo para a formação de professores.

Algumas estratégias foram adotadas para o sucesso do programa, como: o site ETCC, projetado e desenvolvido pelo EBHC, serviu como uma plataforma para acelerar o aprendizado colaborativo entre a comunidade e-professor; todos os conteúdos dos eprofessor foram mantidos, dessa forma, esses conteúdos podiam ser consultados ainda que após a competição, servindo como modelo para outros professores de como construir um ensino colaborativo; e a premiação para equipe vencedora.

[Lin et al. 2011], analisaram o processo após 3 anos do programa ETCC. Segundo os autores, a vantagem é que cada professor do time e-professor, pode promover suas habilidades profissionais, se tornar um aprendiz ao longo da vida e transformar toda a comunidade e-professor. Ainda de acordo com os autores, para que exista um ensino colaborativo é preciso se ter uma base robusta, investidores públicos ou privados que garantam a sustentabilidade do programa; e apoio ao corpo docente e discente.

\section{Revisão Sistemática da Literatura}

Segundo [Mafra and Travassos 2005], uma revisão sistemática da literatura deve seguir uma metodologia rigorosa, confiável e passível de auditoria. Além disso, a revisão deve ter bem definidos os protocolos de busca afim de possibilitar que a pesquisa seja repetida por outros pesquisadores. Nos trabalhos de [Keele 2007] e [Brereton et al. 2007], os autores apresentam uma sequência de passos bem definidos de como realizar tal revisão, também utilizados para desenvolvimento deste trabalho.

Na concepção de [Petticrew and Roberts 2008], a fim de se delimitar o escopo da pesquisa e auxiliar no desenvolvimento das suas questões, e da string de busca, optou-se por utilizar a estrutura PICOC que trata em responder mais de uma pergunta na mesma metodologia.

De acordo com os autores [Keele 2007] e [Petticrew and Roberts 2008], a estratégia PICOC trata dos critérios: População (Population): as pessoas ou grupo que se quer estudar; Intervenção (Intervention): o que se está interessado em rever; Comparação (Comparison): a intervenção é comparada com o que; Saída (Outcomes): em muitas intervenções sociais existe um conjunto grande de resultados e com isso a avaliação en- 
volve alguns critérios; e Contexto (Context): quando a metodologia tem mais de uma pergunta na mesma avaliação. Nesta pesquisa não será utilizado o critério saída da estratégica PICOC.

Para [Keele 2007], a estratégia de busca deve permitir que a pesquisa seja repetida por outros pesquisadores. Para que isto seja possível, deve-se divulgar a string de busca utilizada, assim como levar em consideração as diferentes bases de dados analisadas. Segundo [Kitchenham et al. 2006], o elemento essencial ao se fazer uma revisão sistemática é fazer a string de busca, pois ela constitui a cadeia de pesquisa automática, determina os dados extraídos e restringe a busca. Outro fator importante é que a string de busca faz parte do protocolo e a partir do momento que o protocolo é aceito e validado, a string de busca não deve ser alterada.

\subsection{Questão da Pesquisa}

A questão de pesquisa se refere a colaboração do ensino entre professores e foca os meios colaborativos que os professores utilizam. Porém, além da questão da pesquisa principal, uma questão secundária visa investigar se tal colaboração entre os professores conta com o auxílio de software.

Questão principal (QP): Qual o papel para melhorar a colaboração entre professores no desenvolvimento de suas atividades?

Questão secundaria (QS): Quais sistemas são adotados pelos professores para fomentar a colaboração com seus pares durante o desenvolvimento de suas atividades?

\subsection{Processo da Pesquisa}

A pesquisa foi realizada no período selecionado compreendido entre 2009-2015 para buscar o estado da arte na temática do ensino colaborativo entre professores. As bibliotecas digitais escolhidas foram ACM e IEEEXplore. Essa escolha é justificada por essas bibliotecas possuírem publicações de artigos e revistas; bem como possuem publicações relacionadas com a área de sistemas colaborativos e serem bases em ciências da computação.

Se buscou o estado da arte da temática seguindo a revisão sistemática e utilizando a string de busca: ("collaborative learning" OR "collaborative teaching") AND (teacher OR instructor OR educator OR professor) and ("didactic material" OR "educational activity” OR "pedagogical activity” OR courseware).

Foram identificados critérios de inclusão e exclusão dos artigos relevantes ao tema de pesquisa aplicado a string de busca definida, afim de selecionar os trabalhos que tenham relevância para a pesquisa. Os resultados das buscas foram revisados por três pesquisadores envolvidos, e as discordâncias foram discutidas e resolvidas. Em casos de falta de consenso sobre o trabalho estudado o mesmo é incluído.

Os critérios de inclusão adotados neste trabalho foram: estudos que definem colaboração entre professores para o desenvolvimento de suas atividades, bem como estudos que demonstrem o uso de sistemas para colaboração entre professores nas atividades. Os critérios de exclusão adotados neste trabalho foram: estudos onde a cooperação ou colaboração não foram avaliadas, estudos repetidos ou duplicados foi considerado apenas o mais recente, estudos que apresentem texto, conteúdo e resultados incompletos, estudos pagos e estudos em workshops, resumos, cartazes, painéis, palestras e demonstrações. 
A metodologia utilizada para selecionar os artigos foi realizada seguindo a metodologia de [Santos et al. 2012] tanto para os critérios de inclusão/exclusão como para os critérios de qualidade que constam na Tabela 1.

Foram aplicados os critérios de inclusão/exclusão em cada um dos passos para se manter o maior número de artigos relevantes no estado da arte da temática. [Santos et al. 2012] colocam quatro passos a serem seguidos da metodologia, são eles: Passo 1: Eliminação por Título; Passo 2: Eliminação por resumo; Passo 3: Eliminação por leitura diagonal; e Passo 4: Eliminação por leitura completa.

O escopo para aplicação desta RSL, diz respeito a métodos de colaboração entre professores, sejam eles no planejamento de suas aulas, na criação e compartilhamento de atividades, métodos de ensino ou experiências. Para que o estado da arte fosse delimitado aos métodos de avaliação para sistemas colaborativos optou-se em utilizar a metodologia da RSL de [Santos et al. 2012]. Nesta metodologia os autores utilizam 12 anos para se fazer as buscas do estado da arte da temática escolhida. Contudo, a estratégia desta pesquisa se baseia nos períodos de 2009-2015.

[Santos et al. 2012] mencionam que existe muito a ser estudado e validado em sistemas colaborativos; assim como muita coisa está surgindo. Outro ponto abordado pelos autores é a importância de se colocar critérios de qualidade para o método escolhido e seu respectivo peso. Os critérios de qualidade escolhidos para selecionar os artigos relacionados que demonstrem o estado da arte da temática desta pesquisa podem ser visualizados na Tabela 1. Esses critérios serão aplicados no passo 4, leitura completa dos artigos, e após tal seleção eles se encontram na etapa IV da Tabela 2 e de forma detalhada na Tabela 3. Esses critérios são adaptados da RSL de [Santos et al. 2012], assim como os pesos aplicados para seleção dos artigos.

Tabela 1. Critérios de Qualidade adaptados de [Santos et al. 2012]

\begin{tabular}{|l|c|}
\hline Critérios de Qualidade & Peso \\
\hline O estudo define claramente o objetivo da pesquisa (define questão de pesquisa)? & 1 \\
\hline O artigo responde às questões de pesquisa definidas? & 1 \\
\hline O artigo relata os passos do método que foram seguidos para a avaliação? & 2 \\
\hline O artigo cita o contexto do sistema colaborativo em análise? & 2 \\
\hline O artigo apresenta a motivação para a escolha do método? & 1 \\
\hline Apresenta dificuldades para avaliar o sistema colaborativo? & 2 \\
\hline O artigo apresenta o custo/benefício de utilizar o método estudado? & 2 \\
\hline Total & $\mathbf{1 1}$ \\
\hline
\end{tabular}

A Tabela 2 mostra os artigos classificados em cada uma das etapas da RSL. Os artigos foram selecionados por meio da revisão sistemática utilizando a string de busca nas bibliotecas citadas e de acordo com os critérios de eliminação expostos, por ordem de relevância.

Na Etapa 1 os artigos foram eliminados pelo Passo 1: eliminação pelo título, os artigos eliminados na Etapa 2 se deram decorrentes ao Passo 2: eliminação pelo resumo, os artigos eliminados na Etapa 3 ocorreram pelo Passo 3: leitura diagonal. Já para os artigos que foram selecionados na Etapa 4 foram analisados o Passo 4: leitura completa. 
Tabela 2. Artigos Classificados nas Etapas da RSL

\begin{tabular}{|c|c|c|c|c|c|}
\hline Base de Dados & Analisados & Etapa I & Etapa II & Etapa III & Etapa IV \\
\hline $\boldsymbol{A C M}$ & 411 & 46 & 14 & 10 & 2 \\
\hline IEEE Xplorer & 180 & 126 & 80 & 30 & 3 \\
\hline Total & 591 & 172 & 94 & 40 & 5 \\
\hline
\end{tabular}

Após concluir todos os passos foram selecionados, cinco artigos relacionados com o ensino colaborativo entre os professores, conforme mostra a Tabela 2 na Etapa IV. A Tabela 3 contempla os cinco artigos selecionados com alguns detalhes como: sua referência (autores), o contexto que o mesmo está inserido, o meio utilizado no ensino colaborativo entre os professores.

Tabela 3. Referências dos Artigos Selecionados

\begin{tabular}{|c|c|c|c|c|}
\hline ID & Artigos Selecionados & Autor(es) & Contexto & $\begin{array}{l}\text { Meio utili- } \\
\text { zado }\end{array}$ \\
\hline 1 & $\begin{array}{l}\text { A Keyword Recom- } \\
\text { mendation Experiment } \\
\text { to Support Informa- } \\
\text { tion Organization } \\
\text { and Folksonomies in } \\
\text { Edu-AREA }\end{array}$ & $\begin{array}{l}\text { Caeiro- } \\
\text { Rodríguez }\end{array}$ & $\begin{array}{l}\text { Compartilhamento } \\
\text { de atividades de } \\
\text { ensino pela WEB }\end{array}$ & $\begin{array}{l}\text { Software } \\
\text { Colaborativo } \\
\text { (Edu-Area) }\end{array}$ \\
\hline 2 & $\begin{array}{l}\text { A Proposal Model to } \\
\text { Monitor Interdiscipli- } \\
\text { nary Research Projects } \\
\text { in Latin American } \\
\text { Universities }\end{array}$ & $\begin{array}{l}\text { Heredia and } \\
\text { Vinueza }\end{array}$ & $\begin{array}{l}\text { Interdisciplinariedade } \\
\text { (IL) }\end{array}$ & Web Ontology \\
\hline 3 & $\begin{array}{l}\text { Opening Lesson Plans } \\
\text { to Support Teaching } \\
\text { Innovation and Open } \\
\text { Educational Resources } \\
\text { Adoption }\end{array}$ & Rodríguez & $\begin{array}{l}\text { Desenvolvimento e } \\
\text { compartilhamento } \\
\text { de atividades de } \\
\text { ensino }\end{array}$ & $\begin{array}{l}\text { Software } \\
\text { Colaborativo } \\
\text { (Edu-Area) }\end{array}$ \\
\hline 4 & $\begin{array}{l}\text { A Collaborative Envi- } \\
\text { ronment for the Design } \\
\text { of Accessible Educatio- } \\
\text { nal Objects }\end{array}$ & $\begin{array}{l}\text { Boccacci, } \\
\text { Ribaudo and } \\
\text { Mesiti }\end{array}$ & $\begin{array}{l}\text { Ambiente colabora- } \\
\text { tivo para desenvolvi- } \\
\text { mento de material de } \\
\text { ensino }\end{array}$ & VisualPedia \\
\hline 5 & $\begin{array}{l}\text { Open educational re- } \\
\text { sources in computer } \\
\text { science teaching }\end{array}$ & $\begin{array}{l}\text { Dichev and } \\
\text { Dicheva }\end{array}$ & $\begin{array}{l}\text { Recursos educacio- } \\
\text { nais abertos }\end{array}$ & OER \\
\hline
\end{tabular}

\section{Análise dos Resultados}

Após a conclusão das etapas de seleção a qual os artigos foram submetidos, foram obtidos cinco resultados relacionados com o ensino colaborativo.

As avaliações descritas neste artigo têm o intuito de melhorar a colaboração entre professores durante a realização de suas atividades, bem como identificar os sistemas utilizados para que possam melhorar o processo de ensino-aprendizagem. 
A Tabela 3 mostra os artigos selecionados no ensino colaborativo, seus autores, o contexto que está inserido e o(s) meio(s) utilizado(s). Os artigos classificados tratam da QP: "qual o papel para melhorar a colaboração entre professores no desenvolvimento de suas atividades? " e da QS: "quais sistemas são adotados pelos professores para fomentar a colaboração com seus pares durante o desenvolvimento de suas atividades?". As questões específicas foram todas analisadas e respondidas na revisão dos artigos na etapa IV.

Nos artigos 1 e 3, [Caeiro-Rodríguez et al. 2015b] e [Rodríguez 2014] descrevem a ferramenta Edu-Area. Nela, os professores podem criar ou modificar recursos, atividades e planos de aula para o seu contexto de ensino. Além disso, os professores também podem relatar experiências de atividades desenvolvidas com os alunos, podendo anexar fotos, vídeos, etc. Outro fator interessante é que o Edu-Area é uma ferramenta web, e o material disponibilizado é considerado um recurso educacional aberto (OER), permitindo sua utilização, replicação e adaptação quando necessário. Assim, segundo os autores, a ferramenta Edu-Area traz um novo desenvolvimento, apoiando a operação dos usuários de forma aberta e na organização social.

No artigo 2, [Heredia and Vinueza 2015] tratam da colaboração que pode existir dentro de uma universidade, mais em especifico dentro de um curso onde acontece a interdisciplinaridade que pode gerar possíveis inovações na área pesquisada.

No artigo 4, [Boccacci et al. 2009] discorrem acerca de uma ferramenta web com o foco no desenvolvimento de material de ensino de forma colaborativa. Por meio da ferramenta, além de colaborar com o conteúdo do material, os professores contribuem no desenvolvimento de materiais que atendam pessoas com todo tipo de deficiência, como por exemplo, pessoas com deficiência visual necessitam de materiais em forma de áudio, ou pessoas com deficiência auditiva que necessitam de materiais de forma ilustrativa, as imagens utilizadas são tratadas por um algoritmo matemático no VisualPedia; o que pode ser desenvolvido por mais de um professor. A colaboração é unida a necessidade de inclusão das pessoas com diversas formas de deficiência, obtido com a integração da MediaWiki.

No artigo 5, [Dicheva and Dichev 2014] relatam sobre os OERs. Eles afirmam que a busca por materiais de ensino é frequente, porém muitas vezes os conteúdos disponibilizados não atendem ou não se adéquam a realidade do professor, não atendendo as expectativas. Segundo os autores, não é suficiente ter apenas uma grande quantidade de conteúdo, também são necessários critérios de filtro e de busca para que esses conteúdos possam ser facilmente encontrados, porém ainda é baixo o conhecimento existente sobre OER. A pesquisa revela os diferentes pontos de vista dos usuários e das soluções fornecidas. Contudo, conforme os autores, não adianta apenas disponibilizar a informação. Também é preciso apoiar o ensino nas diferentes atividades, nos critérios de seleção, na facilidade de encontrar o desejado e na sua adaptação.

Neste artigo foi realizada uma revisão sistemática com um método consolidado, que especificam, passo a passo o que deve ser realizado. Contudo, o resultado obtido ainda não foi satisfatório. Dos 591 artigos resultantes da busca pelas palavras chaves apenas cinco destes foram selecionados como pertinentes ao estado da arte da temática. Assim, o resultado encontrado nos indica que a busca deve ser realizada em mais bibliotecas digitas, 
não somente as bases de ciências da computação. Os artigos em português também devem ser incluídos, bem como os critérios de exclusão devem ser revistos, como por exemplo, a exclusão por título.

\section{Discussões}

Após a análise dos resultados, vista na seção anterior, as pesquisas relacionadas com os 5 artigos selecionados por meio da RSL foram estendidas com o intuito de complementar o estudo sobre a colaboração durante o processo de ensino-aprendizagem.

Nos artigos 1 e 3, [Caeiro-Rodríguez et al. 2015b] e [Rodríguez 2014] relatam sobre a ferramenta Edu-Area. Conforme [Bergman et al. 2008], quando uma organização categoriza a informação, melhora-se o acesso a informação, sua pesquisa e a sua recuperação por futuros usuários. Não apenas quem categoriza tem a informação, mas toda a comunidade de usuários. Segundo [Caeiro-Rodríguez et al. 2015a], não houve uma adoção da ferramenta Edu-Área, pois houveram problemas e barreiras no processo de sua adoção. Os principais problemas encontrados foram decorrentes da divergência do apoio efetivamente realizado do que foi proposto; e as diferentes abordagens ao conceito de organização e usuários.

No artigo 2, [Heredia and Vinueza 2015] analisam a colaboração que pode existir dentro de uma universidade, especificamente em disciplinas multidisciplinares. Os autores [Khalili et al. 2007] em pesquisas anteriores, mencionam que as equipes multidisciplinares precisam de tempo para que os alunos possam colocar seu projeto em uso e o testar em toda sua extensão. Outro ponto é o incentivo para o corpo docente, avaliações trimestrais para que os ajustes do curso possam ocorrer durante o mesmo e não apenas no final das atividades. Outra consideração é que para os alunos contemplarem as diferenças culturais, sociais e econômicas precisam aprender uma segunda língua, tais como espanhol ou inglês. Segundo [Ktoridou and Eteokleous 2013], ao incluir alunos de diferentes disciplinas, os alunos são mais inclinados a terem uma perspectiva integrada e as soluções encontradas são oriundas dessa multidisciplinaridade. Os alunos que vem de disciplinas diferentes acabam beneficiando a própria aprendizagem interdisciplinar (IL). Uma vantagem educacional dos programas com abordagem IL é excluir o fracionamento e as habilidades isoladas, os alunos têm uma motivação e uma adesão no aprendizado. Já os professores nesse ambiente podem enriquecer seu ensino com o conhecimento adquirido nos diversos cursos.

No artigo 4, [Boccacci et al. 2009] apresentam uma ferramenta web com o foco no desenvolvimento de material de ensino de forma colaborativa. O material foi desenvolvido para atender todo tipo de pessoas, incluso as pessoas com deficiência, sejam elas: cognitivas, visuais e auditivas. O visualPedia, conforme [Valtolina et al. 2012], é um sistema de e-learning multimídia e audiovisual colaborativo. Ele tem por objetivo a inclusão de alunos com deficiência, bem como apoiar os professores na construção dos materiais utilizados de forma personalizada. O sistema possui um controle de perfis configurável de acordo com as necessidades dos alunos. O processo de concepção e desenvolvimento da ferramenta VisualPedia é analisado em 3 pontos da usabilidade e acessibilidade: avaliação de usabilidade semiótica, análise da acessibilidade técnica e experiência do usuário. De acordo com os autores, nessa abordagem consegue-se produzir sistemas mais utilizáveis e acessíveis; e como trabalhos futuros precisam corrigir os problemas de acessibilidade 
e usabilidade ainda existentes, assim como encontrar novas estratégias de colaboração para construção dos materiais. A ideia para construir esses objetos educacionais é envolver os alunos no preparo desses materiais, pois ninguém mais do que eles sabem quais os métodos que mais se adéquam as suas necessidades, principalmente aqueles que possuem alguma deficiência, resultando no aumento da comunidade; e explorando a inserção dos usuários no uso das ferramentas sociais. Conforme os autores é preciso conquistar o usuário para que ele traga suas habilidades das redes sociais e trabalhe de forma ativa no melhoramento da ferramenta.

No artigo 5, [Dicheva and Dichev 2014] abordam alguns recursos educacionais abertos (OERs). Os autores, em pesquisas anteriores [Dichev and Dicheva 2012], relatam que a forma como os recursos são produzidos, a sua reutilização e distribuição é que são novos. Em decorrência disso, a quantidade de material existente é razoável, o problema é que boa parte desse material não é utilizada. A principal barreira é a sua findability, ou seja, localizar onde está o repositório e o conteúdo dentro do repositório. Para tal, foi mudado a forma como são realizadas as pesquisas de busca, por meio da utilização de um vocabulário específico. Segundo [Dicheva and Dichev 2015], ainda não se tem conhecimento das experiências de OER e por quem eles são utilizados, dessa forma não se sabe o impacto real dos OERs na aprendizagem das disciplinas. Dessa forma, se busca saber quem utiliza e como os OERs são utilizados quando apoiados por uma interface de busca que possibilite a exploração dos mesmos. Os usuários usam o conteúdo, quando sabem da existência dos seus repositórios e de uma ferramenta que permita explorar esse conteúdo. Interfaces que possibilitem uma maior interação resulta num maior engajamento dos usuários. Os autores afirmam que não adianta disponibilizar os OERs, se não houver: divulgação do repositório e de seus objetos, um mapeamento de quem vai utilizálos e apoio aos mesmos; e por último, uma ferramenta de exploração com uma interface amigável e voltada para as necessidades dos usuários.

\section{Conclusões}

Este artigo apresenta uma revisão sistemática da literatura (RSL) de estratégias de ensino colaborativo entre professores. A revisão considerou o período selecionado correspondido entre 2009 a 2015. Inicialmente, 591 artigos foram resultantes da busca. Contudo, após seguir os passos subsequentes da RSL, que se deram em quatro etapas, obteve-se cinco artigos que contemplam o estado da arte da temática.

Os primeiros resultados encontrados demostram algumas iniciativas relacionadas com o ensino colaborativo. Contudo, a principal constatação é que ainda existe muito a ser explorado, tanto em ferramentas que facilitem a colaboração entre os professores, quanto em atividades de que ressaltem para esses profissionais as vantagens da colaboração.

A RSL se mostrou interessante como um ponto de partida para a pesquisa, contudo, acreditamos que é importante complementá-la com busca de trabalhos relacionados com os encontrados na RSL. Assim, a identificação das citações relevantes encontradas nos artigos de encontrados na RSL, bem como a pesquisa por trabalhos que citam tais artigos permite refinar o resultado da pesquisa.

Como trabalhos futuros pretende-se complementar a revisão sistemática da literatura expandindo as buscas para outras bibliotecas digitais, assim como revisar e aprimorar a string de busca, complementando com os novos termos encontrados nos trabalhos rela- 
cionados. Além disso, é importante que a busca seja realizada em mais bibliotecas digitas, não somente as bases de ciências da computação, bem como incluir artigos em português.

Também pretendemos investigar como está funcionando a colaboração entre o professor titular da disciplina e o intérprete ou segundo professor, para os casos dos alunos com deficiências auditivas. Assim, desejamos investigar a colaboração desses profissionais que auxiliam o professor da disciplina na preparação dos materiais de ensinoaprendizagem.

\section{Referências}

Bergman, O., Beyth-Marom, R., and Nachmias, R. (2008). The user-subjective approach to personal information management systems design: Evidence and implementations. J. Am. Soc. Inf. Sci. Technol., 59(2):235-246.

Boccacci, P., Ribaudo, M., and Mesiti, M. (2009). A collaborative environment for the design of accessible educational objects. In Proceedings of the 2009 IEEE/WIC/ACM International Joint Conference on Web Intelligence and Intelligent Agent Technology - Volume 03, WI-IAT '09, pages 207-210, Washington, DC, USA. IEEE Computer Society.

Brereton, P., Kitchenham, B. A., Budgen, D., Turner, M., and Khalil, M. (2007). Lessons from applying the systematic literature review process within the software engineering domain. J. Syst. Softw., 80(4):571-583.

Caeiro-Rodríguez, M., Llamas-Nistal, M., Fernández-Iglesias, M., Mikic-Fonte, F., and Lama-Penín, M. (2015a). Supporting real open educational resources in edu-area: Different views about open educational resources. In Frontiers in Education Conference (FIE), 2015. 32614 2015. IEEE, pages 1-8.

Caeiro-Rodríguez, M., Santos-Gago, J. M., Lama, M., and Llamas-Nistal, M. (2015b). A keyword recommendation experiment to support information organization and folksonomies in edu-area. IEEE Revista Iberoamericana de Tecnologias del Aprendizaje, 10(2):60-68.

Damiani, M. (2008). Entendendo o trabalho colaborativo em educação e revelando seus benefícios. Educar em Revista, 0(31).

Dichev, C. and Dicheva, D. (2012). Open educational resources in computer science teaching. In Proceedings of the 43rd ACM technical symposium on Computer Science Education, pages 619-624. ACM.

Dicheva, D. and Dichev, C. (2014). Finding open educational resources in computing. In Advanced Learning Technologies (ICALT), 2014 IEEE 14th International Conference on, pages 22-24. IEEE.

Dicheva, D. and Dichev, C. (2015). Cs/it oer: Availability, discoverability and use. In Proceedings of the 16th Annual Conference on Information Technology Education, SIGITE '15, pages 133-138, New York, NY, USA. ACM.

Engeström, Y. (1994). Teachers as collaborative thinkers: Activity-theoretical study of an innovative teacher team. Teachers' minds and actions: Research on teachers' thinking and practice, pages 43-61. 
Fullan, M., Hargreaves, A., and Garcez, R. (2000). A escola como organização aprendente: buscando uma educação de qualidade. Biblioteca Artmed : fundamentos da educação. Artmed.

Gately, S. E. and Gately, F. J. (2001). Understanding coteaching components. Teaching exceptional children, 33(4):40-47.

Heredia, R. M. and Vinueza, P. C. (2015). A proposal model to monitor interdisciplinary research projects in latin american universities. IEEE Revista Iberoamericana de Tecnologias del Aprendizaje, 10(3):102-108.

Keele, S. (2007). Guidelines for performing systematic literature reviews in software engineering. In Technical report, Ver. 2.3 EBSE Technical Report. EBSE.

Khalili, N. R., Jacobius, T. M., Acevedo, J., and Nadal, E. O. (2007). Education without borders: Development of an interdisciplinary project based course between iit and itesm.

Kitchenham, B., Mendes, E., and Travassos, G. H. (2006). A systematic review of crossvs. within-company cost estimation studies. In Proceedings of the 10th international conference on Evaluation and Assessment in Software Engineering, pages 81-90. British Computer Society.

Ktoridou, D. and Eteokleous, N. (2013). Interdisciplinary web-based learning practices in higher education. In Global Engineering Education Conference (EDUCON), 2013 IEEE, pages 536-539.

Lin, C.-D. and Lin, M.-J. (2010). Promoting elementary school teachers' professional development by e-teacher community competition. In Educational and Information Technology (ICEIT), 2010 International Conference on, volume 1, pages V1-135V1-139.

Lin, C. D., Lin, M. J., and Zhang, X. S. (2011). Can we transform the e-teacher community to be a learning organization to promote school teachers' professional development. In Communication Software and Networks (ICCSN), 2011 IEEE 3rd International Conference on, pages 245-249.

Mafra, S. N. and Travassos, G. H. (2005). Técnicas de leitura de software: Uma revisão sistemática. XIX Simpósio Brasileiro de Engenharia de Software, SBES, 5.

Nono, M. A. and MIZUKAMI, M. d. G. (2001). Aprendendo a ensinar: futuras professoras das séries iniciais do ensino fundamental e casos de ensino. Reunião anual da ANPED, 24.

Petticrew, M. and Roberts, H. (2008). Systematic reviews in the social sciences: A practical guide. John Wiley \& Sons.

Pugach, M. C. and Johnson, L. J. (1989). The challenge of implementing collaboration between general and special education. Exceptional Children, 56(3):232-235.

Rabelo, L. C. C. (2012). Ensino colaborativo como estratégia de formação continuada de professores para favorecer a inclusão escolar. Universidade Federal de São Carlos.

Rodríguez, M. C. (2014). Opening lesson plans to support teaching innovation and open educational resources adoption. In Proceedings of The International Symposium on Open Collaboration, OpenSym '14, pages 47:1-47:4, New York, NY, USA. ACM. 
Sa'don, N. F., Alias, R. A., and Ohshima, N. (2014). Nascent research trends in moocs in higher educational institutions: A systematic literature review. In Web and Open Access to Learning (ICWOAL), 2014 International Conference on, pages 1-4.

Sa'don, N. F., Alias, R. A., and Ohshima, N. (2016). In Proceedings of the EUROPEAN STAKEHOLDER SUMMIT on experiences and best practices in and around MOOCs, pages $173-184$.

Santos, N. S., Ferreira, L. S., and Prates, R. O. (2012). An overview of evaluation methods for collaborative systems. In Collaborative Systems (SBSC), 2012 Brazilian Symposium on, pages 127-135.

Thurler, M. and Wolff, J. (2001). Inovar no interior da escola. Artmed.

Torres, P. L. (2015). Metodologias para a produção do conhecimento: da concepção à prática. Curitiba : SENAR - PR.

Valtolina, S., Barricelli, B. R., Mesiti, M., and Ribaudo, M. (2012). User-centered design of e-learning tools for users with special needs: The visualpedia case study. $\operatorname{IxD} \& A$, $13: 47-55$. 